\title{
PAPER
}

\section{Potentially reversible conditions in 1000 consecutive memory clinic patients}

\author{
A Hejl, P Høgh, G Waldemar
}

J Neurol Neurosurg Psychiatry 2002;73:390-394

See end of article for
authors' affiliations
......................
Correspondence to:
Professor G Waldemar,
Memory Disorders
Research Unit, Department
of Neurology N6702, The
Neuroscience Centre,
Copenhagen University
Hospital, Rigshospitalet, 9,
Blegdamsvej,
2100-Copenhagen,
Denmark; gunwal@rh.dk
Received
27 December 2001
In revised form 26 April
2002
Accepted 8 May 2002

\begin{abstract}
Objectives: To investigate the prevalence and classification of potentially reversible conditions in a prospective memory clinic cohort of younger and elderly patients with cognitive symptoms.

Patients: 1000 consecutive patients referred during a period of 54 months to a university hospital multidisciplinary memory clinic based in neurology.

Methods: All patients were referred for diagnostic evaluation and treatment of cognitive symptoms. The multidisciplinary staff prospectively established a standardised consensus report for each patient based on the results of clinical and ancillary investigations with classification of cognitive profile, primary underlying cause, and concomitant conditions.

Results: The mean age of the patients was 66.1 years (range 17-98) and 43\% met diagnostic criteria for dementia. A potentially reversible primary aetiology for cognitive symptoms was identified in $19 \%$ and a potentially reversible concomitant condition in $23 \%$ of all patients. In the subgroup of patients with dementia, $4 \%$ had a potentially reversible primary aetiology. Careful clinical examination, routine laboratory tests, and cranial computed tomography identified most of these conditions.

Conclusions: Reversible conditions are most often encountered in patients with mild cognitive disturbances. Although treatment may not always result in full reversal of cognitive symptoms, potentially reversible conditions should be identified in the diagnostic evaluation of the patient.
\end{abstract}

7 he prevalence of dementia in the elderly population (age $\geqslant 65$ years) in Europe is $6.4 \%$, with Alzheimer's disease being the most frequent cause of dementia.

The early symptoms of Alzheimer's disease comprise increased forgetfulness that interferes with the ability to maintain a job or household, difficulties in finding words, loss of initiative and interest, and decreased judgement. However, these symptoms are not specific for Alzheimer's disease. They may occur in many other conditions, including potentially reversible conditions. With the advent of pharmaceutical treatment for Alzheimer's disease, early diagnosis and treatment have become more important, and more patients are referred to physicians for diagnostic evaluation of mild cognitive symptoms.

The management of patients with cognitive or behavioural symptoms may be complex and crosses the boundaries of several medical specialties. In recognition of the need for multidisciplinary assessment and treatment of patients with cognitive symptoms, memory clinics are now being established in many countries. ${ }^{2-3}$ They facilitate early diagnostic assessment, classification, treatment, and follow up of patients with cognitive symptoms and possible dementia in an outpatient setting. It is obvious that not all memory clinic patients have dementia. ${ }^{4}$ Some present with mild cognitive impairment not meeting the criteria for dementia or with other conditions associated with cognitive symptoms. Patients with symptoms that are sufficiently severe to fulfill diagnostic criteria for dementia only rarely have an underlying condition that is fully reversible.

Reversible conditions may occur more frequently in patients referred for mild cognitive complaints. A wide range of studies have analysed the prevalence of potentially reversible conditions in patients with dementia. ${ }^{5-7}$ However, most studies were retrospective and dealt with populations of patients with an established clinical diagnosis of dementia.

To our knowledge the prevalence of potentially reversible conditions in patients referred for cognitive symptoms to a multidisciplinary memory clinic has not previously been described in a prospective setting.

The aim of this study was investigating the prevalence and classification of potentially reversible conditions in a memory clinic cohort. We defined a potentially reversible condition as a condition known to be (a) potentially reversible or arrestable either on treatment or spontaneously, and (b) responsible for, or contributing to, the observed cognitive symptoms or dementia.

\section{METHODS}

\section{Patients}

The memory clinic at the Copenhagen University Hospital was established in September 1995. This report describes the diagnostic evaluation of the first 1000 consecutive patients who were referred and had completed the diagnostic evaluation during a period of 54 months (September 1995 to February 2000). All patients presented with cognitive symptoms.

\section{Diagnostic assessment}

The clinic is an outpatient memory clinic based in neurology. The staff is multidisciplinary with neurologists, psychiatrists, neuropsychologists, neurosurgeons, specialist nurses, and social advisors. Patients are primarily referred by general practitioners and private practice specialists in neurology and psychiatry in Copenhagen. Some patients are referred from other hospitals in the Copenhagen district, as well as from

Abbreviations: CT, computed tomography; DSM-IV, Diagnostic and statistical manual of mental disorders, fourth edition; MMSE, mini-mental state examination; MRI, magnetic resonance imaging; NINCDS-ADRDA, National Institute of Neurological and Communicative Disorders and Stroke-Alzheimer's Disease and Related Disorders Association; NINDS-AIREN, National Institute of Neurological Disorders and Stroke-Association Internationale pour la Recherche et l'Enseignement en Neurosciences; SPECT, single photon emission computed tomography 


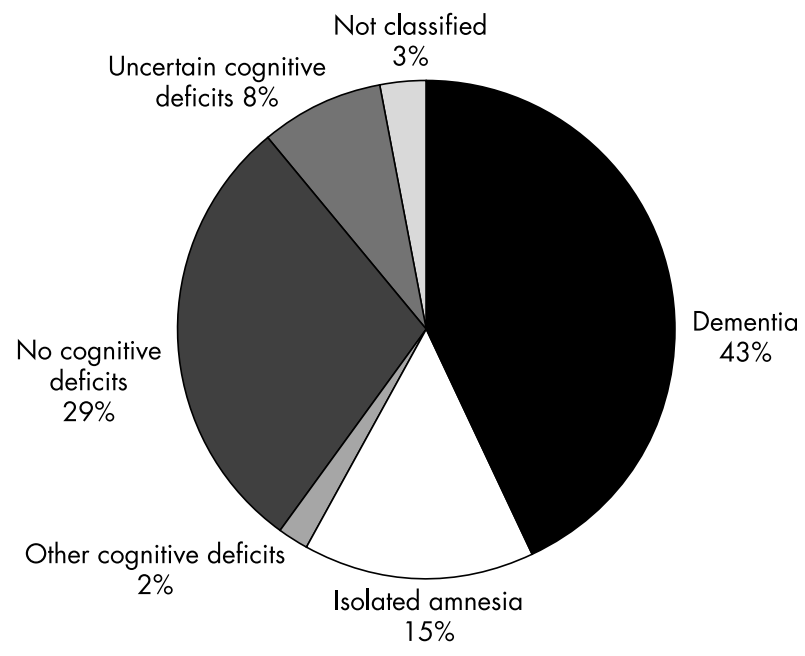

Figure 1 Classification of cognitive profiles $(n=1000)$. Distribution of patients with dementia, selective amnesia, other cognitive deficits, no cognitive deficits, uncertain test data or "not classified" according to consensus panel decision and relevant clinical criteria.

other Danish counties. In particular, patients with suspected normal pressure hydrocephalus are referred from a larger district. On the first visit a neurologist interviewed each patient and the caregiver. In all patients, a physical and neurological examination, laboratory screening tests (blood sedimentation rate, complete blood cell count, electrolytes, glucose, renal and liver function tests, thyroid function test, serum lipids, vitamin $\mathrm{B} 12$, and serological tests for syphilis), and electrocardiography were performed. In addition, global cognitive functions were assessed with the mini-mental state examination (MMSE), ${ }^{8}$ a short cognitive screening test with a maximum score of 30. The neurologist determined the indications for further investigations in collaboration with the other specialists. Most patients had cranial computed tomography (CT) or magnetic resonance imaging (MRI), functional brain imaging with single photon emission computed tomography (SPECT), and a neuropsychological examination. In addition, some patients had electroencephalography and a psychiatric evaluation. Other supplemental tests were performed, if clinically relevant.

The standardised neuropsychological examination program was described previously. ${ }^{9}$ Briefly, the patients underwent an extensive battery of neuropsychological tests covering six classical cognitive domains: memory, concentration and attention, abstraction and problem solving, language, visual perception, and visuoconstruction. In each cognitive domain a composite score was compared with normative data.

After completion of the initial study program, the multidisciplinary staff established a standardised consensus report for each patient. These were reported at weekly conferences in which all specialists on staff participated. The consensus report contained a classification of the cognitive profile, the primary underlying cause, and concomitant conditions.

Firstly, the cognitive profiles of the patients were classified into the following six groups: (a) dementia fulfilling the criteria of the Diagnostic and statistical manual of mental disorders, fourth edition (DSM-IV) ${ }^{10}$; (b) isolated amnesia, not fulfilling dementia criteria; (c) other cognitive deficits; (d) no significant cognitive deficits; (e) uncertain test data; or (f) not classified. Isolated amnesia was defined as a significantly reduced composite memory score and normal function in all other domains on neuropsychological testing. Some patients were classified in the subgroup "uncertain test data". This group comprised patients with mild cognitive symptoms, questionable cognitive deficits not fulfilling dementia criteria, or cognitive "deficits" due to poor cooperation on testing or concomitant psychiatric symptoms. Some patients were not classified according to the system mainly because of language problems or ongoing abuse of alcohol or drugs.

Secondly, the primary underlying aetiology of the cognitive profile was established. For a diagnosis of Alzheimer's disease, the National Institute of Neurological and Communicative Disorders and Stroke-Alzheimer's Disease and Related Disorders Association (NINCDS-ADRDA) criteria ${ }^{11}$ were used. For a diagnosis of vascular dementia, the criteria of the National Institute of Neurological Disorders and Stroke-Association Internationale pour la Recherche et l'Enseignement en Neurosciences (NINDS-AIREN) ${ }^{12}$ were applied. All other diagnoses were classified according to the International statistical classification of diseases and related health problems, tenth revision. ${ }^{13}$ Patients in whom no specific underlying condition was identified were classified as having "no neuropsychiatric disease".

Thirdly, concomitant conditions, which may contribute to the cognitive symptoms, were registered.

In some patients a decision concerning diagnostic classification and treatment could not be made at the first consensus conference. These patients were discussed again at a future conference after obtaining more information or performing additional investigations.

Systematic data on diagnostic assessments were collected prospectively. For primary and concomitant conditions all

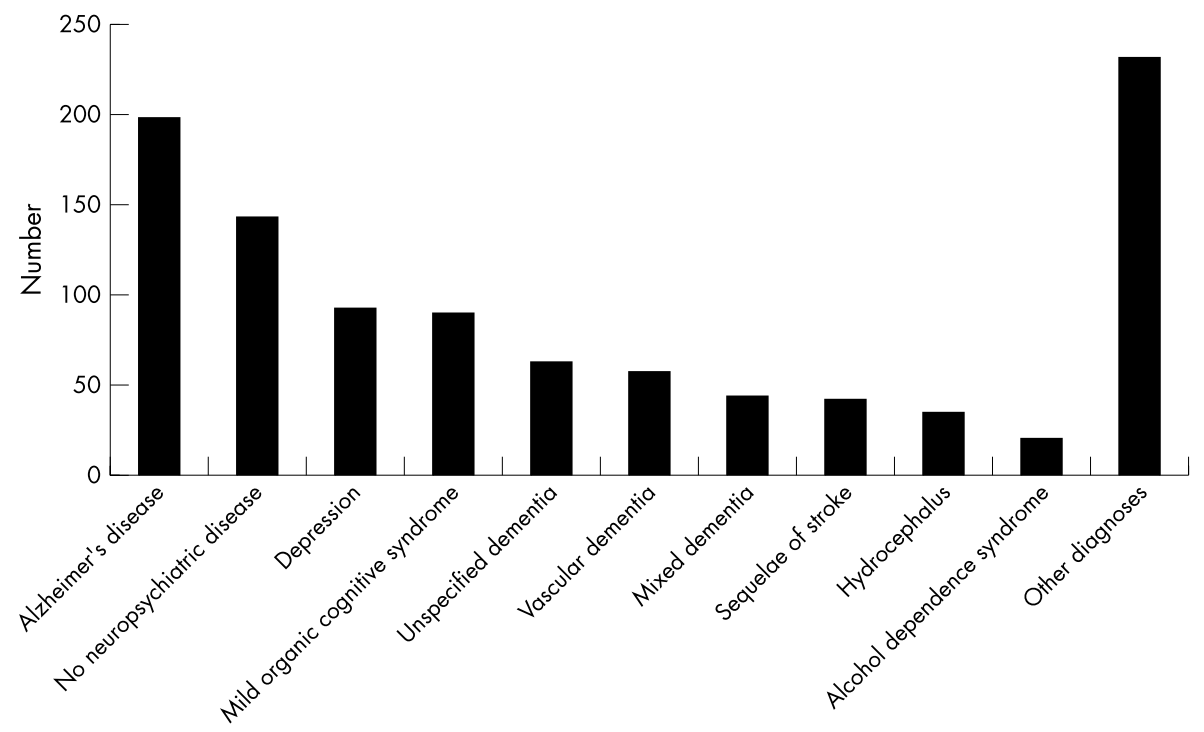

Figure 2 Final (primary) diagnosis in all patients $(n=1000)$. Distribution of the 10 most frequently applied primary diagnoses. The primary diagnosis was the primary underlying cause of the presenting cognitive symptoms according to the consensus panel decision and relevant clinical criteria. 
Table 1 Prevalence of potentially reversible underlying causes and of potentially reversible concomitant conditions in all 1000 patients and in subgroups of patients

\begin{tabular}{|c|c|c|c|}
\hline & Number & $\begin{array}{l}\text { Potentially reversible primary } \\
\text { underlying cause (\%) }\end{array}$ & $\begin{array}{l}\text { One or more potentially reversible } \\
\text { concomitant conditions }(\%)\end{array}$ \\
\hline All patients & 1000 & 19 & 23 \\
\hline \multicolumn{4}{|l|}{ Grouped by cognitive profile } \\
\hline Dementia & 432 & 4 & 23 \\
\hline Objective cognitive deficits but not dementia & 170 & 16 ** & 31 * \\
\hline No objective cognitive deficits & 286 & 38 ** & 23 \\
\hline \multicolumn{4}{|l|}{ Grouped by age and sex } \\
\hline Age $\geqslant 60$ years & 685 & 14 & 22 \\
\hline Men & 297 & 16 & 24 \\
\hline Women & 388 & 13 & 20 \\
\hline Age $<60$ years & 315 & 28 ** & 26 \\
\hline Men & 176 & 27 & 23 \\
\hline Women & 139 & 29 & 29 \\
\hline
\end{tabular}

potentially reversible conditions were registered. Patient characteristics and diagnostic classifications were registered consecutively in a database.

\section{Statistical analyses}

The prevalence of potentially reversible conditions was determined in the total group of patients as well as for the following subgroups: patients who fulfilled dementia criteria; patients with cognitive symptoms not fulfilling dementia criteria; patients with no objective cognitive deficits; and younger (age $<60$ years) and elderly patients (age $\geqslant 60$ years). In each age group possible differences between sexes were analysed. The prevalences in the subgroups were compared using $\chi^{2}$ two tailed tests. The level of significance was set at $p<0.05$.

\section{RESULTS}

A total of 1000 consecutive patients (473 men and 527 women) with memory problems, other cognitive symptoms, or possible dementia were referred and completed the diagnostic evaluation during a period of 54 months. Their mean age was 66.1 years (range 17-98) and their mean MMSE score was 23.1 (range 0-30). Most of the patients were referred by general practitioners $(58 \%)$ or private practice specialists in neurology and psychiatry (12\%). The remaining patients $(30 \%)$ were referred from other hospitals in the Copenhagen district and other Danish counties. Most of the patients (89\%) had structural brain imaging (CT or MRI), 67\% had functional

Table 2 Potentially reversible primary aetiologies for cognitive symptoms in 1000 memory clinic patients

\begin{tabular}{ll}
\hline Diagnosis & $\begin{array}{l}\text { Patients with potentially } \\
\text { reversible causes }(n=185)\end{array}$ \\
\hline Depression & 98 \\
Hydrocephalus & 34 \\
Alcohol dependence syndrome & 19 \\
Space-occupying lesions & 8 \\
Metabolic diseases & 8 \\
Epilepsy & 7 \\
Post-traumatic syndromes & 6 \\
Obstructive sleep apnoea & 4 \\
Delirium & 1 \\
\hline
\end{tabular}

Hydrocephalus comprised normal pressure hydrocephalus and other types of hydrocephalus. Intracranial tumours and vascular malformations were grouped as space-occupying lesions. Metabolic diseases were hyperthyroidism, hypothyroidism, and one case of porphyria. Post-traumatic syndromes were whiplash sequelae and post-traumatic stress disorder. brain imaging (SPECT), 53\% had a neuropsychological examination, and $26 \%$ had psychiatric evaluation.

\section{Classification of cognitive profiles and final diagnoses}

Figure 1 shows the distribution of cognitive profiles into the six categories: $43 \%$ fulfilled criteria for dementia, $15 \%$ for isolated amnesia, and 2\% for other cognitive deficits, and 29\% had no cognitive deficits. The remaining patients were not classified.

Figure 2 presents the primary aetiological diagnoses in the 1000 patients. The 10 most frequent causes of the presenting cognitive symptoms were seen in $77 \%$ of all patients. The remaining patients had a wide range of other neurological, psychiatric, or internal medicine diseases. As shown, $14 \%$ of all patients were classified with "no neuropsychiatric disease". This diagnosis was more frequent in the younger patients below 60 years $(29 \%)$ than in the older patients $(7 \%)$.

\section{Potentially reversible conditions}

The prevalence of a potentially reversible primary underlying cause was $19 \%$. Table 1 shows the prevalence in subgroups of patients. Potentially reversible primary causes were significantly more frequent in the younger than in the older patients, and significantly more frequent in patients with subjective cognitive symptoms only than in patients with an established diagnosis of dementia. No significant difference was observed between sexes.

Table 2 lists the potentially reversible conditions. The three most common primary potentially reversible diagnoses, present in $82 \%$ ( 151 of 185 patients) of all patients with potentially reversible conditions, were depression, hydrocephalus, and alcohol dependence syndrome. Excluding patients with hydrocephalus who were referred from a larger district, $15 \%$ had a potentially reversible primary cause.

Of all 1000 patients, 432 fulfilled the DSM-IV criteria for dementia. In this subgroup, $18(4 \%)$ had a potentially reversible primary cause. Hydrocephalus was seen in the majority of these patients (15 patients), while three patients had space-occupying lesions.

Potentially reversible or arrestable concomitant conditions with possible relevance to the presenting cognitive symptoms were identified in $23 \%$ of all 1000 patients. The five most frequent conditions were depression $(8 \%)$, thyroid disease $(4 \%)$, B12 deficiency (4\%), alcohol dependence syndrome (3\%), and epilepsy $(2 \%)$. For concomitant conditions, potentially reversible causes were significantly more frequent in patients with cognitive deficits than in those with dementia. There were no significant differences between the age groups. 


\section{DISCUSSION}

To our knowledge this is the first study to investigate potentially reversible conditions in a prospective cohort of younger as well as older memory clinic patients. In 1000 consecutive patients we found a potentially reversible aetiology in $19 \%$ and a potentially reversible concomitant condition in $23 \%$. Among patients with symptoms meeting the criteria for dementia, a potentially reversible primary aetiology was less frequent and observed in only $4 \%$. We found the highest prevalence $(38 \%)$ in the subgroup of patients with self reported memory complaints, which were not verified on neuropsychological testing. A medium high prevalence (16\%) was found in the subgroup of patients with cognitive deficits not meeting dementia criteria. In general, the prevalence was higher in the younger patients $(28 \%)$ than in the older patients (14\%).

The most frequently encountered potentially reversible conditions were depression, normal pressure hydrocephalus, alcohol related conditions, space-occupying lesions, epilepsy, and metabolic conditions.

The prevalence of potentially reversible conditions in dementia was studied in several smaller studies, most of which were summarised in two major reviews. In a meta-analysis of 32 studies including 2889 patients, Clarfield ${ }^{5}$ found a frequency of $13.2 \%$ for potentially reversible conditions. In 1995, Weytingh et al published a quantitative review of 16 studies, in which they found that the frequency of potentially reversible dementia varied widely from 0 to $37.5 \%$, with an average of $15.2 \%$.

The prevalence found in our prospective study was higher than, although within the same range as, in previous studies. However, several factors may influence the prevalence of potentially reversible conditions in patients with possible dementia and explain the variation between studies. Firstly, the referral pattern may vary depending on the setting of the study (neurological, psychiatric, or geriatric; inpatient or outpatient). Thus, memory clinics based in neurology may attract younger patients and more patients with mild cognitive symptoms. Secondly, a study with systematic prospective registration of a structured classification of diagnoses may yield more potentially reversible cases than a retrospective study. Thirdly, the definition of potentially reversible conditions may vary between studies. Clarfield ${ }^{5}$ included spaceoccupying lesions, hydrocephalus, depression, infections, metabolic disorders, and drug induced dementia as potentially reversible conditions, whereas Weytingh et al also included alcoholism. In our study, epilepsy, certain posttraumatic syndromes, obstructive sleep apnoea, and delirium were also considered to be potentially reversible. Cognitive function may improve in patients with unrecognised epilepsy and memory complaints after antiepileptic treatment. ${ }^{14}$ Cognitive complaints are often reported by patients suffering from long lasting post-traumatic syndromes. These symptoms tend to improve spontaneously in most patients ${ }^{15}$ but it is important to exclude other aetiologies. Untreated obstructive sleep apnoea may be complicated with mild cognitive symptoms. ${ }^{16}$ Although memory clinic patients rarely present with delirium, it is well known that delirium is associated with impairment of memory and attention, which may resolve on treatment.

Some potentially reversible conditions may not be reversible on treatment in the individual patient. In their quantitative review, Weytingh et al $^{7}$ found that the frequency of partially or fully reversible conditions was lower $(9.3 \%$ and $1.5 \%$, respectively) than that of potentially reversible conditions (15\%). Walstra et al $l^{6}$ reported in a prospective study of 170 elderly patients fulfilling criteria for dementia that the prevalence of potentially reversible concomitant conditions was $19 \%$. Complete reversal of dementia was not observed in any patients. However, although treatment may not always result in full reversal of cognitive symptoms, potentially reversible conditions should be identified as part of the diagnostic evaluation of dementia.

In patients with an established diagnosis of a progressive cognitive disorder, it is important to identify and treat potentially reversible concomitant conditions that may further reduce the functional capacity of the patient. The potentially reversible primary and concomitant conditions identified in this study fall under a wide range of neurological, psychiatric, or internal medicine diagnoses. Therefore, all physicians should be aware of the possibility of potentially reversible conditions in patients with cognitive complaints.

The mix of neurological, psychiatric, and internal medicine aetiologies in dementia and cognitive disorders also underscores the need for a multidisciplinary assessment. Verhey et $a l^{17}$ found that a multidisciplinary approach led to a substantial difference in diagnosis from a monodisciplinary approach. The neurologists often did not report depression, and somatic conditions with relevance for cognitive disorder were often not reported by the psychiatrists.

Most of the reversible conditions in our study were identified easily by careful physical, neurological, and psychiatric examination and history taking, structural brain imaging, or routine laboratory tests. With a prevalence of $3 \%$ for hydrocephalus and $1 \%$ for space-occupying lesions, and with other important diagnostic and treatment implications of cerebrovascular lesions, brain imaging with CT or MRI should be performed in all patients referred for diagnostic evaluation of dementia, also recommended in recent clinical practice guidelines. ${ }^{18-19}$

In conclusion, although potentially reversible conditions may not be fully reversible on treatment, the identification and treatment of these conditions are essential elements of early diagnostic evaluation of dementia, whether the patient is cared for by family physicians or by hospital based specialists. Physicians may advise their patients that not all those with memory impairment have Alzheimer's disease and that treatment may improve or restore intellectual functions. This advice is particularly important for patients with mild cognitive symptoms, as some potentially reversible conditions may progress into irreversible conditions if left untreated. Depression is the most frequent of the potentially reversible conditions and has the highest probability of cure. Most of the potentially reversible conditions are easily identified by careful clinical examination of the patient, routine laboratory tests, and brain imaging.

\section{ACKNOWLEDGEMENTS}

We thank the 1991 Pharmacy Foundation, the Health Insurance Fund, and the IMK Almene Fond for financial support to the development of the memory clinic and associated research projects. We are grateful to clinical nurse Ane Eckermann, Oda Jakobsen, Annette Lauridsen, and Hanne Sørensen for the organisation of patient study programs and for help with registration of data.

\section{Authors' affiliations}

A Hejl, P Høgh, G Waldemar, Memory Disorders Research Unit, Department of Neurology, The Neuroscience Centre, Copenhagen University Hospital, Rigshospitalet, Copenhagen, Denmark

Competing interests: none declared

\section{REFERENCES}

1 Lobo A, Launer L, Fratiglioni L, et al. Prevalence of dementia and major subtypes in Europe: a collaborative study of population-based cohorts. Neurologic Diseases in the Elderly Research Group. Neurology 2000;54:S4-9.

2 Bayer AJ, Pathy MS, Twining C. The memory clinic: a new approach to the detection of early dementia. Drugs 1987;33:84-9.

3 Hogh P, Waldemar G, Knudsen GM, et al. A multidisciplinary memory clinic in a neurological setting: diagnostic evaluation of 400 consecutive patients. Eur J Neurol 1999;6:279-88. 
4 Kopelman M Crawford S. Not all memory clinics are dementia clinics. Neuropsychol Rehabil 1996;6: 187-202.

5 Clarfield AM. The reversible dementias: do they reverse? Ann Intern Med 1988;109:476-86.

6 Walstra GJ, Teunisse S, van Gool WA, et al. Reversible dementia in elderly patients referred to a memory clinic. J Neurol 1997;244:17-22.

7 Weytingh MD, Bossuyt PM, van Crevel $\mathrm{H}$. Reversible dementia: more than $10 \%$ or less than $1 \%$ ? A quantitative review. J Neurol 1995;242:466-71.

8 Folstein MF, Folstein SE, McHugh PR. "Mini-mental state". A practical method for grading the cognitive state of patients for the clinician. $J$ Psychiatr Res 1975;12:189-98.

9 Waldemar G, Bruhn P, Schmidt E, et al. Cognitive profiles and regiona cerebral blood flow patterns in dementia of the Alzheimer type. Eur J Neurol 1994;1:81-9.

10 American Psychiatric Association. Diagnostic and statistical manual of mental disorders, 4th edition. Washington: American Psychiatric Association, 1994

11 McKhann G, Drachman D, Folstein M, et al. Clinical diagnosis of Alzheimer's disease: report of the NINCDS-ADRDA Work Group under the auspices of Department of Health and Human Services Task Force on Alzheimer's Disease. Neurology 1984;34:939-44.
12 Roman GC, Tatemichi TK, Erkinjuntti T, et al. Vascular dementia: diagnostic criteria for research studies. Report of the NINDS-AIREN International Workshop. Neurology 1993;43:250-60.

13 World Health Organization. International statistical classification of diseases and related health problems, tenth revision. Geneva: World Health Organization, 1992.

14 Hogh P, Smith SJ, Scahill RI, et al. Epilepsy presenting as AD: neuroimaging, electroclinical features, and response to treatment. Neurology 2002;58:298-301.

15 Kessels RP, Aleman A, Verhagen WI, et al. Cognitive functioning after whiplash injury: a meta-analysis. J Int Neuropsychol Soc 2000;6:271-8.

16 Kimoff RJ, Cosio MG, McGregor M. Clinical features and treatment of obstructive sleep apnea. Can Med Assoc J 1991;144:689-95.

17 Verhey FR, Jolles J, Ponds RW, et al. Diagnosing dementia: a comparison between a monodisciplinary and a multidisciplinary approach. J Neuropsychiatry Clin Neurosci 1993;5:78-85.

18 Waldemar G, Dubois B, Emre M, et al. Diagnosis and management of Alzheimer's disease and other disorders associated with dementia: the role of neurologists in Europe. Eur J Neurol 2000;7:133-44.

19 Knopman DS, DeKosky ST, Cummings JL, et al. Practice parameter: diagnosis of dementia (an evidence-based review): report of the Quality Standards Subcommittee of the American Academy of Neurology. Neurology 2001;56:1143-53.

\section{HISTORICAL NOTE}

\section{Pituitary tumours and acromegaly (Pierre Marie's disease)}

B ecause physicians of the early nineteenth century were unaware of the endocrine system, acromegaly was regarded as an intrinsic bone disease. Andrea Verga ${ }^{1}$ in 1864 and Vincenzo Brigidi in 1877 reported early macroscopic and microscopic descriptions of pituitary tumours in acromegalic patients. ${ }^{2}$

However, Saucerotte $e^{3}$ reported in 1772 an atypical case in a 39 year old man published in $1801 .{ }^{4}$ Lombroso had in 1869 described the enlarged organs and limbs as macrosomia. Major also relates a case with an enlarged pituitary gland at autopsy reported before Marie's paper by Klebs, and Fritzsche in 1884.

Marie published his pathological studies in two patients in $1886,{ }^{5}$ and gave his name to the disorder; he mentions Saucerotte's patient. This was followed by longer communications in the Nouvelle Iconographie de la Salpetrière, and in Brain in 1890. By this time, 38 examples had been reported.

In September 1885 Pierre Marie ${ }^{6}$ proposed the term acro-megalie.

"The two cases were observed in Professor Charcot's clinic. Case I. Fusch, a woman, aged 37 single ... .At the age of twenty-four, following great fatigue and exposure in washing a house, her menstruation suddenly ceased. . . She remained in bed three weeks; there was no pain in the joints, but she noticed, on raising herself, a weakness of the left hand, with tingling sensations in it .... From this time she went for three months without menstruating.

"...she was troubled with violent pains, sometimes over the forehead, the parietal eminences, or the temples. . . It was at the age of twenty four, at the time the menstruation suddenly ceased, that she noticed the sudden increase in her hands. Her face at this time also underwent changes, ...so that when the patient returned home none of her relatives could recognize her.

"Present condition. The whole feet are large, including the toes. Though the latter are increased in size, they have preserved their form, there is no true deformity, their appearance is simply that of a very big person.

"The hands are very large, but of regular form; their thickness and width are relatively greater than their length, and attention is at once attracted to them on seeing the patient. . . . There is apparent atrophy of the interosseous muscles, which, according to the account of the patient, took place early in the disease. Movements of the fingers are well performed. . . . The thyroid cartilage seems increased in size, but the thyroid gland is found with difficulty.

"The tongue is enlarged. The patient is a little deaf, and the sight is also slightly defective... The cranial vertex is of nearly the same size as the end of the chin. The lower jaw is well developed. . . The patient's thirst is intense, obliging her to beg tea of her friends in order to satisfy it. The quantity of urine is excessive; . . .there being no sugar."

His second patient, also a female, was aged 54 years. Menstruation had ceased abruptly when she was 29 . At 30 she completely lost her eyesight. After eight months she recovered some vision but after two years, she became permanently blind. From the age of 32, though previously referred to as "la petite," her waist, hands, and feet were getting bigger. Excessive thirst was another symptom. Marie described her "acro-megalie" in detail.

How to identify this strange symptom puzzled Pierre Marie and his chief, Charcot. He found five similar case reports (Saucerotte (1772); Alibert (1822); Friedreich, two cases (1868); and Henrot (1877, 1882)). In Henrot's case, at postmortem an ovoid tumour the size of a hen's egg was found near the pituitary body. This finding anticipated Minkowski's demonstration of pituitary enlargement in acromegaly. In $1887^{7}$ Marie recognised the important association with pituitary tumours, and described recognisable features of kyphosis, thirst polyuria, and carpal tunnel syndrome.

A sad postscript is the moving autobiography by Leonard Portal Mark, MD, himself a sufferer. ${ }^{8}$ His friends had noticed signs in his early twenties, but it was not until he was 49 when

"during [a] French medical visit to London I was spotted in a crowd by Dr Pierre Marie as a typical acromegalic..

Post tenebras lux!"

J M S Pearce

304 Beverley Road, Anlaby, Hull HU10 7BG, UK jmspearce@freenet.co.uk

\section{References}

1 Verga A. R.C. Real Inst. Lombardo 1864; vol 3. In: Wilson SAK. Neurology Vol 2. London: Edward Arnold, 1940: 1254.

2 Brunori A, Bruni P, Delitala A, et al. Acromegaly and pituitary tumors: early anatomoclinical observations. Surg Neurol 1995;44:83-7.

3 Garison \& Morton's Medical Bibliography, 5th edn. Edited by Jeremy M Norman. Aldershot: Scolar Press 1991: 605 (note 3880).

4 Saucerotte $\mathbf{N}$. Accroisement singulier en grosseur des os d'un homme agé de 39 ans. Mélanges de chirugie Paris 1801;1:407-11.

5 Pierre M. Sur deux cas d'acromegalie. Hypertrophe singuliere no congénitale des extrémités supérieures, inférieures et cephalique. Révue Medicale Française 1886;6:297-333.

6 Pierre M. Two cases of acromegaly. Translated by Proctor S Hutchinson. London: New Sydenham Society, 1891. In: Major RH. A History of Medicine Volume 2. Oxford: Blackwell, 1954:305-7.

7 Minkowski O. Ueber einen Fall von Akromegalie. Berlin klin Wschr 1887;24:371-4

8 Mark LP. Acromegaly. A personal experience. London: Baillière, Tindall and Cox, 1912 